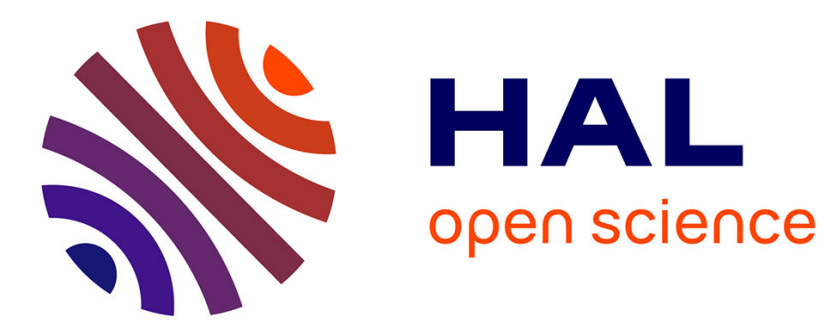

\title{
L'accélérateur linéaire à électrons du C. E. N. de Saclay E. Picard
}

\section{- To cite this version:}

E. Picard. L'accélérateur linéaire à électrons du C. E. N. de Saclay. J. Phys. Radium, 1956, 17 (7), pp.600-601. 10.1051/jphysrad:01956001707060000 . jpa-00235490

\section{HAL Id: jpa-00235490 https://hal.science/jpa-00235490}

Submitted on 1 Jan 1956

HAL is a multi-disciplinary open access archive for the deposit and dissemination of scientific research documents, whether they are published or not. The documents may come from teaching and research institutions in France or abroad, or from public or private research centers.
L'archive ouverte pluridisciplinaire HAL, est destinée au dépôt et à la diffusion de documents scientifiques de niveau recherche, publiés ou non, émanant des établissements d'enseignement et de recherche français ou étrangers, des laboratoires publics ou privés. 


\title{
L'ACCÉLÉRATEUR LINÉAIRE A ÉLECTRONS DU C. E. N. DE SACLAY
}

\author{
Par E. PICARD, \\ Centre d'Études Nucléaires, Saclay.
}

\begin{abstract}
Sommaire. - On décrit rapidement l'accélérateur linéaire d'électrons en construction au Centre d'Études Nucléaires de Saclay. Le courant moyen du faisceau accéléré est égal à $85 \mu \mathrm{A}$ pour une énergie de $28 \mathrm{MeV}$, et un cycle utile de $1 / 1000$. Le faisceau, en tombant sur une cible d'uranium, crée un flux moyen de $10^{13}$ neutrons par seconde. Une déflexion magnétique sélectionne un faisceau d'électrons monochromatique dont la dispersion relative en énergie est de l'ordre de $10^{-3}$. L'équipement expérimental d'utilisation est décrit.
\end{abstract}

Abstract. - The linear electron accelerator, which is being built at the Centre d'Etudes Nucleaires of Saclay, is briefly described. The main caracteristics of the beam are a mean current $85 \mu \mathrm{A}$ for an energy of $28 \mathrm{MeV}$, and a duty cycle of $1 / 1000$. The beam, when falling on an uranium-target, creates a mean flux of $10^{13}$ neutrons per seconde. An accurate magnetic deflection provides a monochromatic electron beam, the relative spread of which will be of the order of $10^{-3}$. The experimental facilities are described.

La construction de l'accélérateur linéaire a débuté en mai 1955. L'appareil doit être terminé pour la fin de l'année 1956 ; études et réalisation auront donc duré environ 18 mois.

Les caractéristiques principales de l'accélérateur linéaire sont données dans le tableau ci-dessous.

Caractéristiques de l'accélérateur linéaire à électrons. Énergie : $28 \mathrm{MeV}$ (correspondant au courant max.).

Courant max : $85 \mathrm{~mA}$ crête.

Durée des impulsions : $0,25-0,5-1-2 \mu \mathrm{s}$.

Fréquence de répétition : $500-250-125$ pps.

Énergie variable de 10 à $33 \mathrm{MeV}$ (une extension est prévue portant à $45 \mathrm{MeV}$ l'énergie du faisceau).

La puissance $\mathrm{HF}$ nécessaire à l'accélération du faisceau correspond à un rendement de $30 \%$

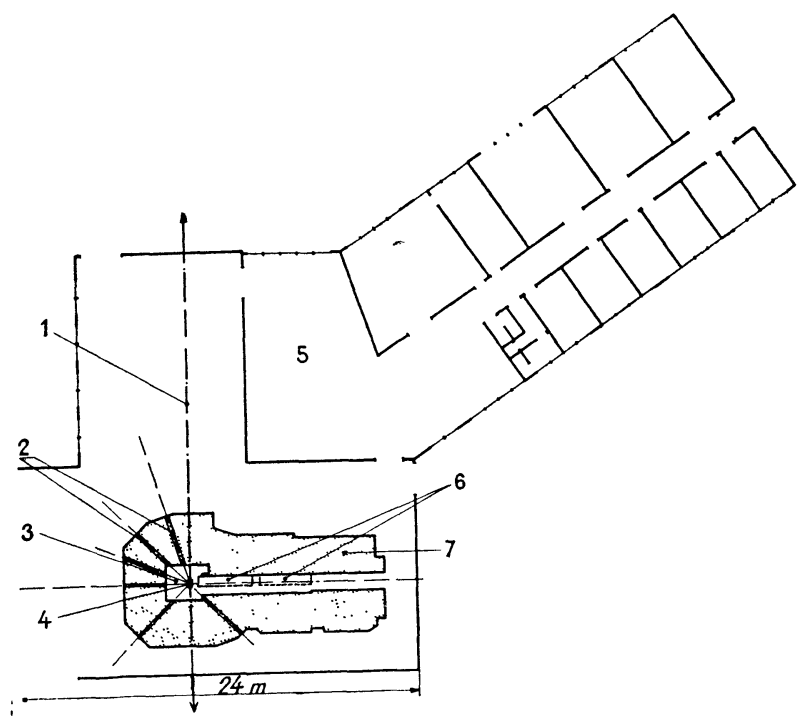

Fig. 1a. - 1) Départ de la base de temps de vol ; 2) canaux d'utilisation ; 3) chambre des cibles ; 4) cible ; 5) salle de contrôle ; 6) tables support de l'accélérateur ; 7) protection en béton. environ, soit $2,4 \mathrm{~kW}$ cédés au faisceau sur une puissance $\mathrm{HF}$ moyenne égale à $8 \mathrm{~kW}$.

L'implantation de la machine est visible dans la figure 1. La figure $1 a$ situe l'accélérateur et son massif de protection dans le bâtiment prévu. La figure $1 b$ représente la tête de l'accélérateur.

Le flux de neutrons émis par une cible en uranium placée dans le faisceau d'électrons doit avoir une intensité moyenne de $10^{13}$ neutrons/s., soit $10^{16}$ neutrons/s. durant l'impulsion. Cette source de neutrons permettra d'effectuer des mesures par la méthode du temps de vol dans des domaines d'énergie beaucoup plus étendus que précédemment et avec des résolutions meilleures. L'intensité atteinte rend possible l'utilisation d'une base dont

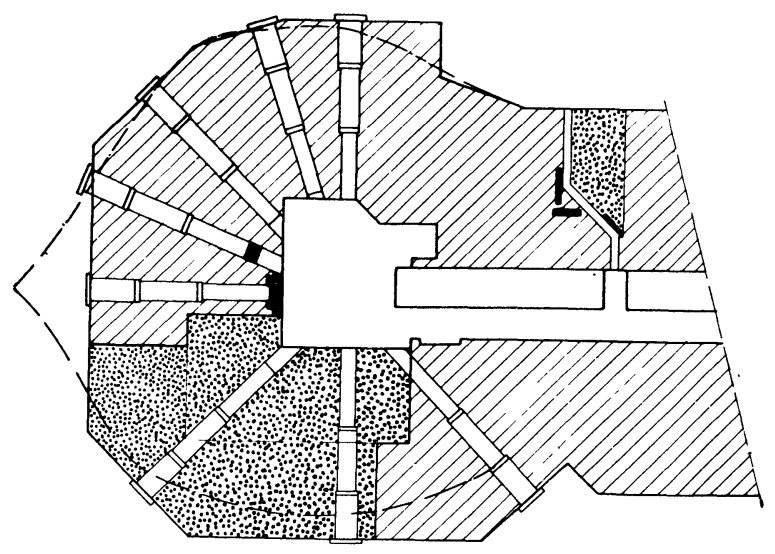

FIg. 1b. - Coupe horizontale à la hauteur du faisceau du massif de protection entourant la chambre des cibles. En pointillé, la partie de la protection effectuée en, dalles; en hachures, la partie massive de béton et en noir des briques de plomb incorporées dans le béton. Sur la figure on distingue les différents canaux horizontaux d'exploitation. La courbe en traits mixtes représente l'intensité du niveau $\gamma$ en l'absence de plomb incorporé dans le béton. 
la longueur atteint 200 mètres. Par exemple pour des neutrons de $1 \mathrm{MeV}$, le temps de vol est de 14 microsecondes environ, permettant une résolution $\frac{\Delta E}{E}$ meilleure que $10 \%$. Deux bases de temps de vol, dont les départs sont visibles sur la figure $1 a$, sont utilisées simultanément. Des stations intermédiaires sont prévues sur leur parcours. Les sélecteurs de temps de vol sont situés dans la salle de contrôle.

Dans la chambre des cibles ( $f g .1 b$ ) se trouve un monochromateur qui est constitué de deux déflecteurs magnétiques à $60^{\circ}$. Ce monochromateur sélectionnera ceux des électrons du faisceau accéléré dont l'énergie est égale, à $0,5 \%$ oo près, à une valeur choisie entre 10 et $33 \mathrm{MeV}$. Le faisceau monochromatique d'électrons est environ 40 fois plus intense que celui d'un bétatron. Ce faisceau sera utilisé par l'action des photons secondaires pour des mesures de spectrométrie nucléaire à moyenne énergie (détermination d'énergie propre de noyaux, largeur des niveaux, sections efficaces d'absorption....), des expériences de diffusion inélastique des électrons sur les noyaux et enfin des mesures sur la polarisation du faisceau de freinage.

Un appareillage de haute précision est en cours de réalisation pour ce type d'expérience.

Des irradiations d'échantillons pourront être effectuées grâce à des tubes s'approchant très près de la cible. En particulier 2 tubes pneumatiques relieront la chambre des cibles à une salle d'expérimentation, ce qui permet l'examen d'un échantillon un temps très bref après son irradiation. Divers canaux ( $f g .1 b$ ) sont prévus. Dans l'un d'entre eux, le faisceau de particules rayonnées comportera un minimum de $\gamma$ pour un niveau de neutrons non altéré. Ceci a été réalisé en utilisant la directivité marquée vers l'avant du rayonnement $\gamma$, alors que le flux de neutrons émis par la cible est très sensiblement isotrope. 